\title{
Pour un même paysage, deux articles à croiser :
}

\section{Les conceptions normatives du paysage. \\ Le cas des Grands Causses}

Jacques Lepart, Pascal Marty, Olivier Rousset

\section{Faut-il qu'un paysage soit ouvert ou fermé ? L'exemple de la pelouse sèche du causse Méjan}

Claudine Friedberg, Marianne Cohen, Nicole Mathieu

Dans les politiques de protection et de restauration de la nature, le paysage tient de plus en plus de place. II est pensé comme l'instrument pertinent pour que les stratégies de ceux qui agissent sur la nature, agriculteurs, pasteurs et sylviculteurs en particulier, se conforment aux nouvelles normes sociales. Prenant appui sur une même entité géographique, les Grands Causses dont fait partie le causse Méjan, deux analyses sont publiées en parallèle.

La première pose le problème des fluctuations historiques des conceptions normatives depuis le xixe jusqu'à nos jours et démonte les étapes du passage de référents écologiques à des référents culturels.

Toujours sur la base de cette confrontation entre différents registres de références mais en en élargissant la gamme, la seconde s'attache à la période récente pour montrer l'ambiguilté des conceptions et des stratégies qui se cachent derrière le consensus actuel sur les valeurs attribuées à ce paysage.

Toutes deux ont en commun le croisement des regards disciplinaires et la volonté de préciser les rôles des différents acteurs qu'ils soient scientifiques, gestionnaires ou éleveurs. Dans l'article de Lepart et al., il s'agit surtout des deux premiers. Dans celui de Friedberg et al., s'ajoutent les points de vue des acteurs locaux, éleveurs et touristes. L'intérêt de rapprocher ces deux textes est qu'ils portent sur le même espace géographique, qu'ils traitent de la même question et que, bien que partant de deux démarches différentes et non concertées - et d'une certaine manière complémentaire -, ils arrivent à des conclusions similaires, qui mettent en lumière le caractère hautement hypothétique de la mise en œuvre des politiques de conservation de la nature.

L'analyse des jeux et enjeux autour de la notion de paysage le montre ; elle pose une question de portée générale. 